\title{
Option Pricing under the CEV Model in a Composite- diffusive Regime
}

\author{
Zhidong Guo ${ }^{1}$ and Yunliang Zhang, ${ }^{2, *}$ \\ ${ }^{1}$ College of Mathematics, AnQing Normal University, AnQing 246000, China \\ ${ }^{2}$ College of Mathematics, East China University of Technology, NanChang 330013, China \\ ${ }^{*}$ Corresponding author
}

\begin{abstract}
Constant elasticity of variance model for option pricing in a composite-diffusive regime is established. We obtain the Black-Scholes differential equation and the corresponding Black-Scholes formula for the prices of European call option. Furthermore, we discuss an asymptotic expansion of the European call option price as the elasticity factor tends to 2 .
\end{abstract}

Keywords-pricing; CEV model; stable subordinator; asymptotic expansion

\section{INTRODUCTION}

The constant elasticity of variance (CEV, in short) model was introduced by[1, 2]. This model assumes that the dynamics of underlying stock price is governed by

$$
d S_{t}=\mu S_{t} d t+\sigma S_{t}^{\beta / 2} d B_{t}
$$

where $B_{t}$ is a standard Brownian motion, $\mu$ is the rate of mean return of the stock, $\sigma$ is the volatility, $\beta$ is the elasticity factor. When $\beta=2$ the $\mathrm{CEV}$ model degenerates to the classical Black-Scholes (BS, in short) model [3]. Empirical evidence (see [4-6]) has shown that the CEV diffusion process could be a better candidate for describing the actual stock price behaviour than the BS model. Moreover, The CEV model can capture implied volatility's smile or skew phenomena while the classical BS model cannot.

In [7], Magdziarz applied the subdiffusive mechanism of trapping events to describe properly financial data exhibiting periods of constant values and introduced the subdiffusive geometric Brownian motion (SGBM, in short)

$$
X_{\alpha}(t)=X\left(S_{\alpha}(t)\right)
$$

as the model of asset prices exhibiting subdiffusive dynamics. Here, $X_{\alpha}(t)$ is a subordinated process, in which $X(\tau)$ is the Geometric Brownian Motion (GBM, in short) and $S_{\alpha}(t)$ is the inversed $\alpha$ stable subordinator defined in the following way

$$
S_{\alpha}(t)=\inf \left\{\tau>0: U_{\alpha}(\tau)>t\right\}, 0<\alpha<1,
$$

where $U_{\alpha}(\tau)$ is a strictly increasing $\alpha$-stable Levy process (see,[8,9]).Based on the work of Magdziarz, scholars([11-14]) generalize the subdiffusuve Brownian motion model.Specially, Liang et al.[10] generalize Magdziarz's model to a compositediffusive regime. They introduced a composite-diffusive geometric Brownian motion

$$
X_{\alpha, H}(t)=X_{H}\left(S_{\alpha}(t)\right)
$$

as the model of asset prices, where $X_{H}(\tau)$ is the Fractional Geometric Brownian Motion (FBM, in short).

In this paper, based on the work of Magdziarz [7] and Liang et al.[10], we will study the option pricing problem under the $\mathrm{CEV}$ model in a composite-diffusive regime, i.e., the underlying stock price $Z_{t}=X_{H}\left(S_{\alpha}(t)\right)$ satisfies the following stochastic differential equation

$$
d Z_{t}=\mu Z_{t} d S_{\alpha}(t)+\sigma Z_{t}^{\beta / 2} d b_{H}\left(S_{\alpha}(t)\right),
$$

where $\mu, \sigma, \beta, Z_{0}$ are constants, and

$$
d b_{H}(\tau)=\omega(\tau)(d \tau)^{H}
$$

is a modified fractional Brownian motion with Hurst exponent $H \in\left[\frac{1}{2}, 1\right], \omega(\tau)$ is the companion Gaussian white noise with zero mean and unit variance. In particular, it is assumed that the $S_{\alpha}(t)$ is independent of $b_{H}(t)$.

In addition, Beckers[6] finds thirty-seven out of forty-seven stocks in a year daily data set to have estimated $\beta$ to be less than 2. So, in this paper, we assume that $0 \leq \beta<0$.

The rest of the paper is organized as follows. In section 2, we obtain a Black-Scholes equation driven by $X_{H}\left(S_{\alpha}(t)\right)$ defined by equation (2) and give the corresponding BlackScholes formula for the option price of an European call option 
for $\alpha H>\frac{1}{2}$. In Section 3, by using the perturbation theory (see, [15]) for PDE, we obtain an asymptotic representation of the European call option price as the elasticity factor $\beta$ tends to 2 .

\section{BLACK-SCHOLES EQUATION AND FORMULA}

In this section, we follow the other usual assumptions of the classical BS model but with the exception that the underlying stock price satisfies (2).

Let $C\left(t, S_{t}\right)$ be the value at time $t$ of a European call option with expiration $T<+\infty$ and exercise price $K$. We have the following theorem.

Theorem 1 Assume that the price of option $C\left(t, Z_{t}\right)$ belongs to $C^{1,2}([0, T) \times[0,+\infty))$, then $C\left(t, Z_{t}\right)$ satisfies the following partial differential equation

$$
\frac{\partial C}{\partial t}+\frac{H \sigma^{2}}{\Gamma(\alpha)^{2 H}} t^{2 \alpha H-1} Z^{\beta} \frac{\partial^{2} C}{\partial Z^{2}}+r Z \frac{\partial C}{\partial Z}-r C=0,
$$

with boundary condition

$$
C\left(T, Z_{T}\right)=\left(Z_{T}-K\right)^{+}
$$

where $r$ is the risk-free interest rate.

Proof : By the Taylor's formula, we have

$$
\begin{gathered}
C(t+\Delta t, Z+\Delta Z)=C(t, Z)+\frac{\partial C(t, Z)}{\partial t} \Delta t \\
+\frac{\partial C(t, Z)}{\partial Z} \Delta Z+\frac{1}{2} \frac{\partial^{2} C(t, Z)}{\partial t^{2}}(\Delta t)^{2}+\frac{1}{2} \frac{\partial^{2} C(t, Z)}{\partial Z^{2}}(\Delta Z)^{2} \\
+\frac{\partial^{2} C(t, Z)}{\partial t \partial Z} \Delta t \Delta Z+o\left((\Delta t)^{2}+(\Delta Z)^{2}\right)
\end{gathered}
$$

From [10] we know that

$$
\begin{aligned}
& \Delta Z=\mu Z \frac{t^{\alpha-1}}{\Gamma(\alpha)} \Delta t+\sigma Z \Delta b_{H}\left(S_{\alpha}(t)\right)+o(\Delta t), \\
& (\Delta Z)^{2}=\sigma^{2} Z^{\beta}\left(\frac{t^{\alpha-1}}{\Gamma(\alpha)}\right)^{2 H}+O(\Delta t)^{\min (1,2 H)},
\end{aligned}
$$

$$
(\Delta Z)(\Delta t)=o(\Delta t)
$$

We construct a portfolio $\Pi$ of $C(t, Z)$ and $Z$ such that

$$
\Pi_{t}=C(t, Z(t))-\Delta Z(t)
$$

here, $\Delta=\frac{\partial C(t, Z)}{\partial Z}$ denotes the units of the stock, $\Pi$ is the price of a riskless portfolio. Then, it follows from (8)-(11) that

$d \Pi=\left[\frac{\partial C(t, Z)}{\partial t}+\sigma^{2} H t^{2 H-1}\left(\frac{t^{\alpha-1}}{\Gamma(\alpha)}\right)^{2 H} Z^{\beta} \frac{\partial^{2} C(t, Z)}{\partial Z^{2}}\right] d t$

If the portfolio is riskless during time $t$, then

$$
d \Pi=r \Pi d t=r\left(C-\frac{\partial C(t, Z)}{\partial Z} Z\right) d t
$$

Thus, we can obtain that

$$
\frac{\partial C}{\partial t}+\frac{H \sigma^{2}}{\Gamma(\alpha)^{2 H}} t^{2 \alpha H-1} Z^{\beta} \frac{\partial^{2} C}{\partial Z^{2}}+r Z \frac{\partial C}{\partial Z}-r C=0,
$$

The proof is completed.

In the end of the section, we discuss the corresponding Black-Scholesformula. Denote

$$
H^{\prime}=\alpha H, \hat{\sigma}=\frac{\sigma}{\sqrt{\alpha} \Gamma(\alpha)^{H}}
$$

then (6) changes into

$$
\frac{\partial C}{\partial t}+\frac{H \sigma^{2}}{\Gamma(\alpha)^{2 H}} t^{2 \alpha H-1} Z^{\beta} \frac{\partial^{2} C}{\partial Z^{2}}+r Z \frac{\partial C}{\partial Z}-r C=0,
$$

Theorem 2 Suppose that $H^{\prime}>\frac{1}{2}$, then the solution of problem (13) and (7) ,i.e. the value of a European call option is given by

$$
C(t, Z)=Z \sum_{n=0}^{\infty} \frac{v^{n} \exp (-v)}{\Gamma(n+1)} G(n+1+1 /(2-\beta), \omega)
$$




$$
\begin{gathered}
-K \exp (-r(T-t)) \sum_{n=0}^{\infty} \frac{v^{n+1 /(2-\beta)} \exp (-v)}{\Gamma(n+1+1 /(2-\beta))} G(n+1, \omega) \\
v=\frac{Z^{2-\beta} \exp (r(2-\beta)(T-t))}{2 c(t)}, \omega=\frac{K^{2-\beta}}{2 c(t)} \\
c(t)=\frac{1}{2} H^{\prime} \hat{\sigma}^{2} \int_{0}^{\tau}(T-s)^{2 H^{\prime}-1} \exp (r(2-\beta)(T-s)) d s, \\
\tau=T-t, G(\xi, \omega)=\frac{1}{\Gamma(\xi)} \int_{\omega}^{\infty} s^{\xi-1} \exp (-s) d s .
\end{gathered}
$$

here $\Gamma(\xi)$ is the Gamma function.

From theorem 2, we can see that $c(t)$ cannot be determined analytically, therefore, the above pricing formula can not to be use directly. Hence we turn into find the asymptotic representation of the solution of the PDE problem (13) and (7).

\section{Asymptotic Representation of Price $C(t, Z(t))$}

In this section, we introduce a small positive parameter $\varepsilon$ such that $\beta=2-\varepsilon$, where $0<\varepsilon<<1$. Then, we will obtain an asymptotic representation of the solution of the PDE problem (13) and (7) in the next theorem.

Theorem 3 Suppose the European call option price $C(t, Z)$ which is the solution of (13) and (7) has an asymptotic expansion price such as

$$
C(t, Z)=C_{0}+\varepsilon C_{1}+\varepsilon^{2} C_{2}+\cdots
$$

Then $C_{0}(t, Z)$ with the final condition

$$
C_{0}(t, Z)=(Z-K)^{+}
$$

is given by

$$
C_{0}(t, Z)=Z N\left(d_{1}\right)-K \exp (-r(T-t)) N\left(d_{2}\right)
$$

where

$$
\begin{gathered}
d_{1}=\frac{\ln (Z / K)+r(T-t)+\frac{\sigma^{2}}{2 \alpha \Gamma(\alpha)^{2 H}}\left(T^{2 \alpha H}-t^{2 \alpha H}\right)}{\sqrt{\frac{\sigma^{2}}{2 \alpha \Gamma(\alpha)^{2 H}}\left(T^{2 \alpha H}-t^{2 \alpha H}\right)}}, \\
d_{2}=d_{1}-\sqrt{\frac{\sigma^{2}}{\alpha \Gamma(\alpha)^{2 H}}\left(T^{2 \alpha H}-t^{2 \alpha H}\right)},
\end{gathered}
$$

$$
N(x)=\frac{1}{\sqrt{2 \pi}} \int_{-\infty}^{x} e^{-y^{2} / 2} d y
$$

and each $C_{n}(t, Z)$ with the final condition $C_{n}(t, Z)=0$ is recursively given by

$$
\begin{gathered}
C_{n}(t, Z)=e^{-r(T-t)} F(x, y), \\
F(x, y)=\frac{1}{2 \sqrt{\pi}} \int_{0}^{x} \int_{-\infty}^{+\infty} \frac{\varphi(\xi, \eta)}{\sqrt{x-\xi}} e^{-\frac{(y-\eta)^{2}}{4(x-\xi)}} d \eta d \xi \\
\varphi(x, y):=e^{-r(T-t)} \sum_{k=0}^{n-1} \frac{(-\hat{Z})^{n-k}}{(n-k) !}\left(\frac{\partial^{2} C_{k}}{\partial \hat{Z}^{2}}-\frac{\partial C_{k}}{\partial \hat{Z}}\right), \\
y=\hat{Z}+r(T-t)-\frac{\sigma^{2}}{2 \alpha \Gamma(\alpha)^{2 H}}\left(T^{2 H}-t^{2 H}\right), \\
x=-\frac{\sigma^{2}}{2 \alpha \Gamma(\alpha)^{2 H}}\left(T^{2 H}-t^{2 H}\right), \\
\hat{Z}=\ln Z .
\end{gathered}
$$

Remark 1 In particular, letting $H=1 / 2$ and $\alpha \rightarrow 1$, then Eq.(13) changes to

$$
\frac{\partial C}{\partial t}+\frac{1}{2} \sigma^{2} Z^{\beta} \frac{\partial^{2} C}{\partial Z^{2}}+r Z \frac{\partial C}{\partial Z}-r C=0,
$$

Therefore, the proposed pricing model of the European call option can be treated as an extension of the model in [19].

Proof From $\hat{Z}=\ln Z$,the PDE problem (13) and (7) becomes

$$
C_{t}+\frac{H \sigma^{2}}{\Gamma(\alpha)^{2 H}} t^{2 \alpha H-1} e^{-\varepsilon \hat{z}}\left(C_{\hat{Z} \hat{z}}-C_{\hat{Z}}\right)+r\left(C_{\hat{z}}-C\right)=0
$$

$$
C(T, \hat{Z})=\left(e^{\hat{Z}}-K\right)^{+}
$$

Now, we define the partial differential operator $L_{0}$ by

$$
L_{0}=\frac{\partial}{\partial t}+\frac{H \sigma^{2}}{\Gamma(\alpha)^{2 H}} t^{2 \alpha H-1}\left(\frac{\partial^{2}}{\partial \hat{Z}^{2}}-\frac{\partial}{\partial \hat{Z}}\right)+r\left(\frac{\partial}{\partial \hat{Z}}-\right)
$$

Then, by using Taylor series of $e^{x}$, from (15-16) we can obtain 


$$
\begin{gathered}
L_{0} C_{0}=0, \\
L_{0} C_{1}=\frac{H \sigma^{2}}{\Gamma(\alpha)^{2 H}} t^{2 \alpha H-1} \hat{Z}\left(\frac{\partial^{2} C_{0}}{\partial \hat{Z}^{2}}-\frac{\partial C_{0}}{\partial \hat{Z}}\right), \\
L_{0} C_{n}=g_{n}(t, \hat{Z}), n \geq 1,
\end{gathered}
$$

where

$g_{n}(t, \hat{Z})=-\frac{H \sigma^{2}}{\Gamma(\alpha)^{2 H}} t^{2 \alpha H-1} \sum_{k=0}^{n-1} \frac{(-\hat{Z})^{n-k}}{(n-k) !}\left(\frac{\partial^{2} C_{k}}{\partial \hat{Z}^{2}}-\frac{\partial C_{k}}{\partial \hat{Z}}\right)$

and the relevant final conditions are given by

$$
\begin{aligned}
& C_{0}(T, \hat{Z})=\left(e^{\hat{Z}}-K\right)^{+}, \\
& C_{n}(T, \hat{Z})=0, n \geq 1 .
\end{aligned}
$$

After changing back to the original variables $Z$ from $\hat{Z}$, the solution of (17) with the final condition (19) is given by

$$
C_{0}(t, Z)=Z N\left(d_{1}\right)-K \exp (-r(T-t)) N\left(d_{2}\right),
$$
where

$$
\begin{gathered}
d_{1}=\frac{\ln (Z / K)+r(T-t)+\frac{\sigma^{2}}{2 \alpha \Gamma(\alpha)^{2 H}}\left(T^{2 \alpha H}-t^{2 \alpha H}\right)}{\sqrt{\frac{\sigma^{2}}{2 \alpha \Gamma(\alpha)^{2 H}}\left(T^{2 \alpha H}-t^{2 \alpha H}\right)}}, \\
d_{2}=d_{1}-\sqrt{\frac{\sigma^{2}}{\alpha \Gamma(\alpha)^{2 H}}\left(T^{2 \alpha H}-t^{2 \alpha H}\right),} \\
N(x)=\frac{1}{\sqrt{2 \pi}} \int_{-\infty}^{x} e^{-y^{2} / 2} d y .
\end{gathered}
$$

In addition, to obtain the correction terms $C_{n}$, we apply the following transformation variables

$$
\begin{gathered}
y=\hat{Z}+r(T-t)-\frac{\sigma^{2}}{2 \alpha \Gamma(\alpha)^{2 H}}\left(T^{2 H}-t^{2 H}\right), \\
x=-\frac{\sigma^{2}}{2 \alpha \Gamma(\alpha)^{2 H}}\left(T^{2 H}-t^{2 H}\right),
\end{gathered}
$$

and

$$
F(x, y)=C_{n}(t, \hat{Z}) e^{r(T-t)},
$$

to (18) and (20) yields

$$
\frac{\partial F}{\partial x}-\frac{\partial^{2} F}{\partial y^{2}}=\varphi(x, y)
$$

$$
F(0, y)=0
$$

where

$$
\varphi(x, y):=e^{-r(T-t)} \sum_{k=0}^{n-1} \frac{(-\hat{Z})^{n-k}}{(n-k) !}\left(\frac{\partial^{2} C_{k}}{\partial \hat{Z}^{2}}-\frac{\partial C_{k}}{\partial \hat{Z}}\right) .
$$

According to heat equation theory,the solution to(24-25) and is given by

$$
F(x, y)=\frac{1}{2 \sqrt{\pi}} \int_{0}^{x} \int_{-\infty}^{+\infty} \frac{\varphi(\xi, \eta)}{\sqrt{x-\xi}} e^{-\frac{(y-\eta)^{2}}{4(x-\xi)}} d \eta d \xi,
$$

since each $g_{n}$ is determined by $C_{i} i=0,1 \cdots, n-1$, therefore is determined recursively.

\section{ACKNOWLEDGMENT}

This work was supported by the Natural Science Foundation of Anhui province (No. AQKJ2015B011 and No. KJ2016A428).

\section{REFERENCES}

[1] J. Cox, "Notes on option pricing I: Constant elasticity of variance diffusions,” Working paper, Stanford University, 1975.

[2] J. Cox, S. Ross, "The valuation of options for alternative stochastic process, “Journal of Financial Economics , pp. 145-166,1976.

[3] F. Black, M. Scholes, "The pricing of options and corporate liabilities," Journal of Political Economy, vol. 81, pp. 637-654, 1973.

[4] S. Hauser, B. Lauterbach, "Tests of warrants pricing model: The trading profits perspective,” Journal of Derivatives, pp. 71-79, 1996.

[5] B. Lautervach, P. Schultz, "Pricing warrants: An empirical study of the Black-Scholes model and its alternatives,” Journal of Finance, vol. 45 pp. 1181-1209, 1990.

[6] S. Beckers, "The constant elasticity of variance model and its implications for option pricing," Journal of Finance, vol. 35, pp. 661673, 1980.

[7] M. Magdziarz, "Black-Scholes formula in subdiffusive regime,” Journal of Statistic Physics, vol. 136, pp. 553-564, 2009.

[8] K.I. Sato, "Processes and Infinitely Divisible Distributions," Cambridge University Press, Cambridge, 1999.

[9] I.M. Sokolov, "Levy flights from a continuous-time process,” Physical Review E, vol. 63, 011104, 2000.

[10] J.R. Liang, J. Wang, L.J. Lv, H. Gu, W.Y. Qiu, F.Y. Ren, "Fractional Fokker-Planck equation and Black-Scholes formula in composite- 
diffusive regime,” Journal of Statistic Physics, vol. 146, pp. 205-216, 2012.

[11] J. Wang , J.R. Liang, L.J Lv ., W.Y. Qiu , F.Y. Ren , “Continuous time Black-Scholes equation with transaction costs in subdiffusive fractional Brownian motion regime," Physica A, vol. 391, pp. 750-759 , 2012.

[12] H. Gu, J.R. Liang, Y.X. Zhang, "Time-changed geometric fractional Brownian motion and option pricing with transaction costs," Physica A, vol. 391, pp. 3971-3977, 2012.

[13] Z.D. Guo, H.J. Yuan, "Pricing European option under the time-changed mixed Brownian-fractional Brownian model," Physica A, vol. 406, pp.73-79, 2014.

[14] Z.D. Guo, "Option pricing under the Merton model of the short rate in subdiffusive Brownian motion regime," Journal of Statistical Computation and Simulation, vol. 87, pp. 519-529, 2017.

[15] E.J. Hinch,"Perturbation Methods," Cambridge University Press, Cambridge, 1991.

[16] G. Jumarie, "On the solution of the stochastic differential equation of exponential growth driven by fractional Brownian motion," Applied Mathmatics Letter, vol. 18, pp. 817-826, 2005.

[17] N.H. Chan, C.T. Ng, "Fractional constant elasticity of variance model, IMS lecture notes-monograph series time series and related topics," vol.52, pp. 149-164, 2006.

[18] G. F. Lo, P. H. Yuan, C. H. Hui, "Constant elasticity of variance option pricing model with time-dependent parameters,International," Journal of Theoretical and Applied Finance, vol. 3, pp. 661-674, 2000.

[19] S. H. Park, J. H. Kim, "Asymptotic option pricing under the CEV diffusion,” Journal of Mathematical Analysis and Applications, vol. 375 pp. 490-501, 2011. 